\title{
Non-prescribed antibiotic dispensing practices for symptoms of urinary tract infection in community pharmacies and accredited drug dispensing outlets in Tanzania. A simulated clients approach
}

\section{Pendo M. Ndaki}

Catholic University of Health and Allied Sciences

Martha F. Mushi

Catholic University of Health and Allied Sciences

Joseph R. Mwanga ( $\nabla$ joseph.mwanga@bugando.ac.tz )

Catholic University of Health and Allied Sciences

Eveline T. Konje

Catholic University of Health and Allied Sciences

\section{Stella Mugassa}

Catholic University of Health and Allied Sciences

Msilikale W. Manyiri

Catholic University of Health and Allied Sciences

\section{Stanley M. Mwita}

Catholic University of Health and Allied Sciences

Nyanda E. Ntinginya

National Institute for Medical Research, Mbeya Medical Research Centre

\section{Blandina T. Mmbaga}

Kilimanjaro Clinical Research Institute, Kilimanjaro Christian Medical Centre and Kilimanjaro Christian

Medical University College

\section{Katherine Keenan}

University of St Andrews

Wilber Sabiiti

University of St Andrews

Mike Kesby

University of St Andrews

\section{Alison Sandeman}

University of St Andrews

Fernando B. -Paez

University of St Andrews

Matthew T.G. Holden 
University of St Andrews

Stephen E. Mshana

Catholic University of Health and Allied Sciences

\section{Research Article}

Keywords: Antibiotic, Antibiotic Resistance, Dispensing Practice, Prescription

Posted Date: February 4th, 2022

DOI: https://doi.org/10.21203/rs.3.rs-1159049/v1

License: (c) (1) This work is licensed under a Creative Commons Attribution 4.0 International License. Read Full License 


\section{Abstract \\ Background}

Antibiotic dispensing without prescription is a major determinant of the emergence of Antimicrobial Resistance (AMR) which has impact on population health and cost of healthcare delivery. This study used simulated clients describing UTI like symptoms to explore compliance with regulation, variations in dispensing practices and drug recommendation, and quality of seller-client interaction on the basis of the gender of the client and the type of drug outlets in three regions in Tanzania.

\section{Method}

A total of 672 Accredited Drug Dispensing Outlets (ADDOs) and community pharmacies were visited by mystery clients (MCs). The study was conducted in three regions of Tanzania namely Kilimanjaro (180, $26.79 \%)$, Mbeya $(169,25.15 \%)$ and Mwanza $(323,48.07 \%)$ in March - May 2020. During data collection, information was captured using epicollect5 software before being analyzed using Stata version 13 .

\section{Results}

Overall, $89.43 \%$ (Cl: $86.87 \%-91.55 \%$ ) of drug sellers recommended antibiotics to clients who described UTI like symptoms but held no prescription and $58.93 \%$ were willing to sell less than the minimum recommended course. Female clients were more likely than male to be asked if they were taking other medications ( $27.2 \%$ vs $9.8 \%$ ), or had seen a doctor ( $27.8 \%$ vs $14.7 \%)$, and more likely to be advised to consult a doctor (21.6\% vs $9.0 \%$ ); pharmacies addressed these issues more often than ADDOs ( $17.7 \%$ vs $13.2 \%, 23.9 \%$ vs $16.6 \%, 17.7$ vs $10.9 \%$ respectively). Sellers recommended 32 different drugs to treat the same set of symptoms, only 7 appear in the Tanzanian Standard Treatment Guidelines as recommended for UTI and 30\% were 2nd and 3rd line drugs. ADDO sellers recommended 31 drug types (including 2nd and 3rd line) but had permission to stock only 3 (1st line) drugs. The most commonly suggested antibiotics were Azithromycin (35.4\%) and ciprofloxacin (20.5\%). Azithromycin was suggested more often in pharmacies (40.8\%) than in ADDOs (34.4\%) and more often to male clients (36.0\%) than female (33.1\%).

\section{Conclusion}

These findings support the need for urgent action to ensure existing regulations are adhered to and to promote the continuing professional development of drug sellers at all outlet levels to ensure compliance with regulation, high quality service and better antibiotic stewardship.

\section{Background}


Since 1940, antibiotics (ABs) have been powerful tools in the clinical management of bacterial diseases [1] and while the development of new ABs has slowed, use has increased massively [2]. Between 2000 and 2015 , daily global consumption of $A B s$ increased by over $60 \%$ from 21.1 to 34.8 billion daily defined doses (DDDs) [3]. In 2015 the annual global ABs consumption was an estimated 42 billion and is projected to rise $200 \%$ by 2030 , with the majority of the increase being in low and middle income countries [3]. The high consumption of ABs, particularly when without prescription, is a growing public health concern because it is associated with the development and spread of antimicrobial resistance (AMR) [4]. Such resistance (and the slow development of alternative ABs) means that potentially lifesaving drugs become ineffective. It has been previously estimated that if action is not taken to address this issue, by 2050 there may be 10 million AMR related deaths worldwide, costing 100 trillion USD [5]. While this figure could be underestimated, low and middle income countries (LMICs) are expected to face the greatest excess deaths and highest health care cost [6] in large part because of their poor economies, limited health care systems and high rates of infectious diseases [7]. The current extent of AMR in LMICs is difficult to quantify due to a limited number of available studies [8].

Although the social determinants of the development of AMR are complicated and multi-layered, widespread overuse and/or misuse of $A B s$ in human (and animal) health care is among the main contributing causes of AMR [9]. About $80 \%$ of global antibiotic consumption occurs outside hospital settings [10], with over $50 \%$ of these community-based ABs purchased from community-based drug outlets or informal retailers, often dispensed without prescription [10]. In most nations, ABs are not 'over the counter' (OTC) non-prescription drugs, yet in many LMIC settings they are frequently dispensed in response to client demand or seller suggestion, accelerating $A B$ misuse and overuse [11]. Access to Abs without prescriptions is a major factor in the development of AMR, facilitating self-medication with ABs and their improper use e.g., incorrect dosage [12]. Despite adequate levels of regulations in most LMICs there is a lack of enforcement [13], hence dispensing ABs OTC is common among all types of drug sellers [3]

In Tanzania ABs are prescription only drugs, but OTC ABs dispensing is frequent [14]. As part of a broader 3-country interdisciplinary study on the drivers of AMR in East Africa (Holistic Approach to Unravel Antibacterial Resistance in East Africa - HATUA - consortium) [15] we have previously reported high OTC provision of ABs across our 3 study sites in Tanzania (94.6\%), with very low numbers of sellers asking clinically relevant questions or offering advice related to ABs stewardship (11.75\%) [16]. Using a similar methodology to the one described below, our Phase 1 study determined how sellers responded to a direct request for amoxicillin from a client who did not have a prescription and who initially did not describe their symptoms. We also determined if a client's request for 'a few days' worth' would induce sellers to dispense less than the regulation minimum course; overall, selling without prescription in our Phase 1 study was $93 \%$.

The Phase 1 study demonstrated variation between regions and between urban and rural locations but over $80 \%$ of sellers in each location sold amoxicillin OTC without a prescription. We also compared the practices of the two types of drug outlets (type 1 pharmacies or type 2 pharmacies/Accredited Drugs 
Dispensing Outlets - ADDOs) mandated by the Tanzania Medicine \& Medical Devices Authority (TMDA) [17]. The former are intended to operate under the supervision of a registered pharmacist, and can sell all categories of retail medicines: narcotics and psychotropic substances, prescription only medicine, pharmacy only medicines, over the counter (OTC) medicines, and general sales medicines [18]. ADDOs meanwhile, are supervised by any person with medical education background (e.g nurses, clinicians etc ) who has attended a five weeks' ADDO training course [19] and so can dispense only a more restricted range of drugs, particularly those that are prescription only (e.g. amoxicillin capsule/suspension, benzylpenicillin powder for injections, chloramphenicol eye drops, Trimethoprim/Sulfamethoxazole suspension, doxycycline capsules/tablets, Phenoxymethylpenicillin suspension/tablets and procaine penicillin fortified) [20]. Our study recorded that fewer pharmacies than ADDOs sold amoxycillin OTC in most regions, but in no region did fewer than $77 \%$ of pharmacies do so [16].

The current paper describes Phase 2 of our study. It explores the quality of the seller/client interaction and the range of $A B s$ sold OTC without a prescription to clients presenting with symptoms of UTI (rather than requesting a named $A B$ ). It examines whether sellers are prepared to allow clients to determine the length of drug course, by dispensing $A B s$ in quantities below the minimum course recommended in the Tanzania Standard Treatment Guidelines (TSTGs) [20]. Finally, the paper investigates variations in practices on the basis of the gender of the client, and between types of outlets in Mwanza, Mbeya and Kilimanjaro regions.

\section{Methods}

\section{Study areas and duration}

In March - May 2020 a multicenter cross-sectional community drug outlets survey was conducted in urban and rural areas of three regions of Tanzania, purposively selected to cover South-western, Northeastern, and North-western mainland Tanzania (respectively: Mbeya region, including Mbeya rural and urban districts: Kilimanjaro region, including Moshi rural and urban districts: and Mwanza region, including Sengerema rural district and Nyamagana and llemela urban districts that form Mwanza, Tanzania's second largest city).

\section{Study procedures and sampling}

We conducted a mystery client study, sometimes referred to as a simulated client study, to assess dispensing of practices. Such study designs are commonly used in LMIC settings and minimise observation bias [3]. The full scenario used by the mystery clients (MCs), shown in supplementary material 1, tested the dispensing practices of sellers presented with a client without a prescription, who described symptoms of urinary tract infection. Using everyday language, the MCs presented with low grade fever, painful micturition, urgency and pyuria and only if questioned, reported the symptoms had been coming and going for the past one month and had not responded to amoxicillin treatment. If offered amoxicillin, the MCs asked 'for something stronger' since amoxicillin 'had not worked'. Only if further questioned, MCs reported that they had only taken a few days' worth of amoxicillin. MCs accepted 
whatever recommendation and treatments the seller advised although if a drug was recommended, they made one request to purchase 'only a few days' worth', before then accepting whatever course the seller recommended. If the seller agreed to this request, we recorded that the $\mathrm{MC}$ had received a 'half course'. In pharmacotherapeutic terms, exactly what constitutes a full course of antibiotic would depend on the drug dispensed and is stipulated in the TSTGs [20]. Having established what drug and course a seller recommended, the $\mathrm{MC}$ either bought or declined to purchase, depending on the price.

After withdrawing and before moving to the next premises, MCs used digital tablets or mobile phones and the software Epicollect 5 [21] to record their findings. MCs recorded the questions asked and advice given by the seller, the medications recommended and in cases where drugs were purchased, the price and quantity of tablets sold. In addition, they recorded the GPS of the premises, and made an attempt to observe or subjectively assess compliance with some of the key minimum guidelines mandated by Tanzanian Guidance on drug sales (i.e.: minimum age of seller should be 18 or over, they should wear uniform and ID badge, display evidence of qualification/certification to practice, and the premises should be in good order and good repair [20].

Between March and May 2020, a total of 672 different sellers were visited in Kilimanjaro (180, 26.79\%), Mbeya $(169,25.15 \%)$, and Mwanza $(323,48.07 \%)$. These drug outlets were selected randomly from the near 100\% sample frame generated in phase 1 of the HATUA project in 2019 (621 in Mwanza, 304 in Mbeya and 232 in Kilimanjaro).

\section{Quality control procedures}

Eleven MCs ( 3 females and 8 males) were trained on the study, its objectives, ethics, and protocols and on the mystery client scenario. To ensure a consistent simulation and data standardization MCs rehearsed the scenario and practiced common behavior and responses with lead researchers familiar with clinical and pharmacy settings. Through the surveying period, lead researchers reviewed the data daily, addressing any inconsistencies with the MC concerned, and met with all MCs twice a week for feedback and updating. All MCs were competent final year bachelor of pharmacy students.

\section{Data management and analysis}

Collected data were downloaded from Epicollect5 and analyzed in Stata version 13 (Stata Corp., College Station, TX, USA) [22]. After data cleaning and consistency checks, the results were analyzed using descriptive univariate and bivariate statistics. The data were summarized and results compared across regions, by type of outlet (ADDOs and community pharmacies) and by clients' gender. Chi square or fishers exact was used to measure the strength of association observed between outlet types and clients' gender. We calculated an additive score for each seller based on whether they asked key questions or gave key advice relevant to good AB stewardship. The questions contributing to this score were: "Did the seller..." 
1....probe you and get you to describe the second set of symptoms (e.g., that you had cloudy, smelly, and bloody pee)?

2. ... ask if you had experienced these symptoms before?

3. ....ask if you were taking any other medication?

4. ....ask if you had seen a doctor?

5. ....ask if you had a prescription?

6. ...suggest you did not need any drug treatment?

7. ...recommend that you see a doctor and/or get a prescription?

8. ... at any point mention or advise you about the issue of antibiotic resistance?

Each question was weighted equally and carried 1 mark if the MC marked 'yes', 0 if the MC marked 'no'. This resulted in an index ranging from 0-8 which was used in some analyses.

\section{Results}

A total of 672 drug outlets were visited by MCs during the study (113 pharmacies and 559 ADDOs). Estimated compliance with mandated regulation was high or very high for some operational attributes, less than $2 \%$ of all those serving the MCs were estimated to be younger than 18 years old (see Table 1). Meanwhile, MCs assessed that $75 \%$ of premises in all regions were in 'adequate' condition (on a 3-point scale), with Kilimanjaro having the least in 'poor' and the most in 'excellent' condition. The majority of sellers displayed certificates of qualifications (74\% in Kilimanjaro, $64 \%$ in Mbeya and 59\% in Mwanza). However, the wearing of ID badges was a rare practice (less than $2 \%$ ) across all three regions (Table 1 ).

\section{TABLE 1: Profile of drug sellers and drug outlets visited by region}




\begin{tabular}{|c|c|c|c|c|c|}
\hline \multirow[t]{2}{*}{ Characteristics } & & Kilimanjaro $n=180$ & Mbeya & Mwanza & Overall \\
\hline & & & n (\%) & n (\%) & n (\%) \\
\hline \multirow[t]{2}{*}{ Gender } & Female & 157 (87.22) & $141(83.43)$ & $229(70.90)$ & $527(78.42)$ \\
\hline & Male & $23(12.78)$ & $28(16.57)$ & $94(29.10)$ & $145(21.58)$ \\
\hline \multirow[t]{2}{*}{ Age } & $<18 \mathrm{yrs}$ & $5(2.78)$ & $1(0.59)$ & $1(0.31)$ & $7(1.04)$ \\
\hline & $>=18 \mathrm{yrs}$ & $175(97.22)$ & $168(99.41)$ & $322(99.69)$ & 665 (98.96) \\
\hline \multirow[t]{2}{*}{ Drug outlet level } & ADDO & $161(89.44)$ & $137(81.07)$ & $261(80.80)$ & 559 (83.13) \\
\hline & pharmacy & $19(10.56)$ & $32(18.93)$ & $62(19.20)$ & $113(16.82)$ \\
\hline \multirow[t]{3}{*}{ Organization } & Poor & $1(0.56)$ & $15(8.88)$ & $31(9.60)$ & 47 (6.99) \\
\hline & Adequate & $134(74.44)$ & $136(80.47)$ & $237(73.37)$ & $507(75.45)$ \\
\hline & Excellent & $45(25.00)$ & $18(10.65)$ & $55(17.03)$ & $118(17.56)$ \\
\hline \multirow[t]{2}{*}{ Display certificates } & No & $47(26.11)$ & $64(37.87)$ & $131(40.56)$ & $242(36.01)$ \\
\hline & Yes & $133(73.89)$ & $105(62.15)$ & $192(59.44)$ & 430 (63.99) \\
\hline \multirow[t]{2}{*}{ Seller wore ID } & No & $176(97.78)$ & $168(99.41)$ & 315 (97.52) & 659 (98.07) \\
\hline & Yes & $4(2.22)$ & $1(0.59)$ & $8(2.43)$ & $13(1.93)$ \\
\hline
\end{tabular}

*ADDO, Accredited Drug Dispensing Outlet, ID, Identification (such as a name tag)

\section{Quality of seller- MC interaction}

The quality of interaction between client and seller, measured by questions asked or advice offered by seller, was generally low with $89.7 \%$ of sellers scoring between 1 and 3 on our 8 -point scale and only $10.3 \%$ scoring between 4 and 7 (Table 2). Proportionally, sellers in pharmacies were more likely than those in ADDOs to address any one of the items on the behavior or advice checklist. Critically however, only in a minority of cases were clients asked one of the three most important questions relevant to dispensing ABs for the treatment of UTI or to give the most important advice, i.e.: 'have you seen a doctor' (17.9\%), 'do you have a prescription' (6.1\%) and 'you should see a doctor to get a prescription' $(12.1 \%)$. 
Again, those in pharmacies were proportionately more likely to address these items than those in ADDOs ( 23.9 vs $16.6 \%, 10.6$ vs $5.1 \%$ and $17.7 \%$ vs $10.9 \%$ respectively). However, while pharmacies performed better as a sector than did ADDOs, over three quarters of pharmacies failed to ask these important questions. Proportionally, female clients were more likely to be asked if they were taking other medications ( $27.2 \%$ vs $9.8 \%$ ) or had seen a doctor $(27.8 \%$ vs $14.7 \%)$ and were more often advised to consult a doctor ( $21.6 \%$ vs $9.0 \%)$.

The results on dispensing practice record that a majority of sellers $89.4 \%$ (Cl: $86.9 \%-91.5 \%)$ displayed a willingness to dispense antimicrobials OTC to clients who described UTI like symptoms and held no prescription. No significant difference $(\mathrm{p}=0.304)$ was observed between ADDOs $(90.0 \%)$ and pharmacies $(86.7 \%)$ or between clients' gender, in the propensity to dispense without prescription. In response to the MCs request for only 'a few days' worth' of drugs, two-thirds of sellers were willing to sell a 'half course' to the client, either with $(29.3 \%)$ or without providing further advice (29.6\%). Proportionally more pharmacies than ADDOs insisted that MCs bought a full course (43.4\% v $27.9 \%)$ see Table 2.

Table 2: Antibiotic dispensing practices and quality of seller's interaction with client by outlet level and client's gender 
female

clients

(total

$\mathrm{n}=162$ ) male

clients

(total

$\mathrm{n}=510$ )
Clients

overall

(total

$\mathrm{n}=672$ )
n (\%)
n (\%)
n (\%)
n (\%)
n (\%)

\section{Quality of interaction}

\begin{tabular}{|c|c|c|c|c|c|}
\hline $\begin{array}{l}\text { 1. Asked about past experience of } \\
\text { symptoms }\end{array}$ & $\begin{array}{l}227 \\
(40.61)\end{array}$ & $57(50.44)$ & $73(45.06)$ & $\begin{array}{l}211 \\
(41.37)\end{array}$ & $\begin{array}{l}284 \\
(42.26)\end{array}$ \\
\hline $\begin{array}{l}\text { 2. Asked about taking other } \\
\text { medication }\end{array}$ & $\begin{array}{l}74 \\
(13.24)\end{array}$ & $20(17.70)$ & $44(27.16)$ & $50(9.80)$ & $94(13.99)$ \\
\hline $\begin{array}{l}\text { 3. Asked if client had seen a } \\
\text { doctor }\end{array}$ & $\begin{array}{l}93 \\
(16.64)\end{array}$ & $27(23.89)$ & $45(27.78)$ & $\begin{array}{l}75 \\
(14.71)\end{array}$ & $\begin{array}{l}120 \\
(17.86)\end{array}$ \\
\hline 4. Asked about prescription & $\begin{array}{l}29 \\
(5.19)\end{array}$ & $12(10.62)$ & $9(5.56)$ & $32(6.27)$ & $41(6.10)$ \\
\hline 5. Suggested no need for a drug & $\begin{array}{l}10 \\
(1.79)\end{array}$ & $7(6.19)$ & $1(0.62)$ & $16(3.14)$ & $17(2.53)$ \\
\hline $\begin{array}{l}\text { 6. Suggested client see a } \\
\text { doctor/get prescription }\end{array}$ & $\begin{array}{l}61 \\
(10.91)\end{array}$ & $20(17.70)$ & $35(21.6)$ & $46(9.02)$ & $81(12.05)$ \\
\hline Mentioned AMR & $\begin{array}{l}41 \\
(7.33)\end{array}$ & $22(19.47)$ & $6(3.70)$ & $\begin{array}{l}57 \\
(11.18)\end{array}$ & $63(9.38)$ \\
\hline \multicolumn{6}{|l|}{ Unwilling to dispense } \\
\hline $\begin{array}{l}\text { - Total who did not recommend a } \\
\text { pharmaceutical drug/were } \\
\text { unwilling to sell }\end{array}$ & $\begin{array}{l}56 \\
(10.02)\end{array}$ & $15(13.27)$ & $21(12.96)$ & $50(9.80)$ & $71(10.57)$ \\
\hline \multicolumn{6}{|l|}{ Willing to dispense } \\
\hline $\begin{array}{l}\text { - Willing to sell but only full- } \\
\text { course }\end{array}$ & $\begin{array}{l}156 \\
(27.91)\end{array}$ & $49(43.36)$ & $43(26.54)$ & $\begin{array}{l}162 \\
(31.76)\end{array}$ & $\begin{array}{l}205 \\
(30.51)\end{array}$ \\
\hline $\begin{array}{l}\text { - Willing to sell half-course but } \\
\text { advice full-course }\end{array}$ & $\begin{array}{l}176 \\
(31.48)\end{array}$ & $21(18.58)$ & $55(33.95)$ & $\begin{array}{l}142 \\
(27.84)\end{array}$ & $\begin{array}{l}197 \\
(29.32)\end{array}$ \\
\hline $\begin{array}{l}\text { - Willing to sell half-course } \\
\text { without any question }\end{array}$ & $\begin{array}{l}171 \\
(30.59)\end{array}$ & $28(24.78)$ & $43(26.54)$ & $\begin{array}{l}156 \\
(30.59)\end{array}$ & $\begin{array}{l}199 \\
(29.61)\end{array}$ \\
\hline $\begin{array}{l}\text { Total willing to sell a } \\
\text { pharmaceutical drug }\end{array}$ & $\begin{array}{l}503 \\
(89.98)\end{array}$ & $98(86.73)$ & $\begin{array}{l}141 \\
(87.04)\end{array}$ & $\begin{array}{l}460 \\
(90.20)\end{array}$ & $\begin{array}{l}601 \\
(89.43)\end{array}$ \\
\hline
\end{tabular}

\section{Patterns of antibiotics dispensed}


A total of 601 sellers recommended 33 different antimicrobials (see table 3 ) to treat the same set of UTIlike symptoms described by MCs. The most commonly suggested antimicrobials were Azithromycin (35.4\%) and ciprofloxacin (20.5\%) which belong to the macrolide and quinolone class respectively and both are in the 'watch' category as indicated in table 3. 'Watch class' antimicrobials (which included azithromycin, cefixime, ceftriaxone, ciprofloxacin, clarithromycin, levofloxacin and moxifloxacin) were recommended during $58.7 \%$ of encounters. Antimicrobials from the 'access' category of drugs (which included Amoxicillin, Ampicillin, Cefadroxil, Cephalexin, Doxycycline, metronidazole, Nitrofurantoi, Notfloxacillin, Tetracycline and Trimethoprim/Sulfamethoxazole) were recommended during $26.8 \%$ of encounters.

Table 3: List of antimicrobials sellers were willing to dispense, and WHO AWaRE categories $(\mathrm{N}=601)$ 


\begin{tabular}{|c|c|c|c|c|}
\hline Suggested Drug (s) & $\begin{array}{l}N- \\
601\end{array}$ & $\%$ & $\begin{array}{l}\text { AWaRE } \\
\text { Categories }\end{array}$ & $\begin{array}{l}\text { Drug class (most } \\
\text { recommended drugs only) }\end{array}$ \\
\hline Amoxicillin & 9 & 1.50 & Access & \\
\hline Amoxicillin and metronidazole & 2 & 0.33 & $\begin{array}{l}\text { Access and } \\
\text { Access }\end{array}$ & \\
\hline Amoxicillin/clavulanic Acid & 23 & 3.83 & $\begin{array}{l}\text { Access and } \\
\text { Access }\end{array}$ & Quinolone \\
\hline Ampicillin & 1 & 0.17 & Access & \\
\hline Ampiclox & 9 & 1.50 & - & \\
\hline Azithromycin & 213 & 35.44 & Watch & Macrolide \\
\hline Azithromycin and Ampiclox & 1 & 0.17 & Watch/- & \\
\hline Azithromycin and Cefalexin & 1 & 0.17 & $\begin{array}{l}\text { Watch and } \\
\text { Access }\end{array}$ & \\
\hline Azithromycin and Cefixime & 1 & 0.17 & $\begin{array}{l}\text { Watch and } \\
\text { Access }\end{array}$ & \\
\hline Azithromycin and Ciplofloxacillin & 2 & 0.33 & $\begin{array}{l}\text { Watch } \\
\text { andWatch }\end{array}$ & \\
\hline Azithromycin and Doxycycline & 5 & 0.83 & $\begin{array}{l}\text { Watch and } \\
\text { Access }\end{array}$ & \\
\hline Azithromycin and Metronidazole & 4 & 0.67 & $\begin{array}{l}\text { Watch and } \\
\text { Access }\end{array}$ & \\
\hline Azithromycin and Nitrofurantoin & 1 & 0.17 & $\begin{array}{l}\text { Watch and } \\
\text { Access }\end{array}$ & \\
\hline Cefadroxil capsule & 1 & 0.17 & Access & Cephalosporin \\
\hline Cefixime & 1 & 0.17 & Watch & \\
\hline Ceftriaxone & 2 & 0.33 & Watch & Cephalosporin \\
\hline Cephalexin & 71 & 11.81 & Access & Cephalosporin \\
\hline Chloramphenicol and Metronidazole & 1 & 0.17 & $\begin{array}{l}\text { Access and } \\
\text { Access }\end{array}$ & \\
\hline Ciprofloxacin & 123 & 20.47 & Watch & Quinolone \\
\hline Ciprofloxacin and Doxycycline & 25 & 4.16 & $\begin{array}{l}\text { Watch and } \\
\text { Access }\end{array}$ & \\
\hline Clarithromycin & 2 & 0.33 & Watch & Macrolide \\
\hline $\begin{array}{l}\text { Doxycycline and } \\
\text { Trimethoprim/Sulfamethoxazole }\end{array}$ & 1 & 0.17 & $\begin{array}{l}\text { Watch and } \\
\text { Access }\end{array}$ & \\
\hline
\end{tabular}




\begin{tabular}{|lccll|}
\hline Doxycycline & 47 & 7.82 & Access & Tetracycline \\
\hline Doxycycline and Metronidazole & 3 & 0.50 & $\begin{array}{l}\text { Watch and } \\
\text { Access }\end{array}$ & \\
\hline Flucamox & 7 & 1.16 & - & \\
\hline Levofloxacin & 11 & 1.83 & Watch & Quinolone \\
\hline Metronidazole & 5 & 0.83 & Access & \\
\hline Moxifloxacin & 1 & 0.17 & Watch & \\
\hline Nitrofurantoin & 7 & 1.16 & Access & \\
\hline Norfloxacillin & 5 & 0.83 & Access & \\
\hline Tetracycline & 1 & 0.17 & Access & Tetracycline \\
\hline Trimethoprim/Sulfamethoxazole & 14 & 2.33 & Access & \\
\hline Trimethoprim/Sulfamethoxazole and & 1 & 0.17 & Access/-- & \\
\hline Ampicloxacillin & & & & \\
\hline total & 601 & 100 & & \\
\hline
\end{tabular}

Only 7 of the drugs recommended by sellers were listed in the TSTGs as suitable to treat the UTI symptoms MCs described (see table 4). Cephalexin (1st line treatment) was recommended by $11.1 \%$ of ADDOs and $15.3 \%$ of Pharmacies, while Ciprofloxacin ( $2^{\text {nd }}$ line treatment) was recommended by $21.9 \%$ of ADDOs and $13.3 \%$ of Pharmacies. The differences in the rate of recommendation between different types of sellers was not statistically significant. Levofloxacin ( $2^{\text {nd }}$ line treatment) was more often suggested by pharmacies (5.1\%) than ADDOs (1.2\%) (p-value of 0.008$)$.

The other antimicrobials, including those most commonly suggested by sellers, were not recommend for the treatment of UTI in the TSTGs. Of these, Azithromycin was suggested by $34.4 \%$ of ADDOs and $40.8 \%$ of pharmacies, with no statistically significant difference between outlet levels. Doxycycline was suggested more by ADDOs (9.0\%) than pharmacies $(2.0 \%)$ with a statistically significant different p-value of 0.020 . Although 31 of the 32 drugs were recommended (and in many cases sold) by ADDOs, there were mandated to sell only 3 of them.

Table 4: List of antimicrobials sellers were willing to dispense by outlet level 


\begin{tabular}{|c|c|c|c|c|c|}
\hline Suggested Drug (s) & $\begin{array}{l}\text { ADDO } \\
\text { (Total } \\
n-503) \\
n(\%)\end{array}$ & $\begin{array}{l}\text { Pharmacies } \\
\text { (Total } \\
\text { n-98) } \\
n(\%)\end{array}$ & $\begin{array}{l}\text { Overall } \\
n-601 \\
n(\%)\end{array}$ & $\begin{array}{l}\mathrm{P}- \\
\text { Value }\end{array}$ & $\begin{array}{l}\text { Not } \\
\text { Recommended for } \\
\text { UTI (NR) } \\
\text { \&Treatment Line }\end{array}$ \\
\hline Amoxicillin* & $\begin{array}{l}9 \\
(1.79)\end{array}$ & $0(0.00)$ & $\begin{array}{l}9 \\
(1.50)\end{array}$ & 0.182 & $1^{\text {st }}$ Line \\
\hline Amoxicillin and metronidazole & $\begin{array}{l}2 \\
(0.40)\end{array}$ & $0(0.00)$ & $\begin{array}{l}2 \\
(0.33)\end{array}$ & 0.532 & $1^{\text {st }}$ Line+ NR \\
\hline Amoxicillin/clavulanic Acid & $\begin{array}{l}9 \\
(1.79)\end{array}$ & $14(14.29)$ & $\begin{array}{l}23 \\
(3.83)\end{array}$ & 0.000 & $3^{\text {rd }}$ line \\
\hline Ampicillin & $\begin{array}{l}1 \\
(0.20)\end{array}$ & $0(0.00)$ & $\begin{array}{l}1 \\
(0.17)\end{array}$ & 0.659 & NR \\
\hline Ampiclox & $\begin{array}{l}9 \\
(1.79)\end{array}$ & $0(0.00)$ & $\begin{array}{l}9 \\
(1.50)\end{array}$ & 0.182 & NR \\
\hline Azithromycin & $\begin{array}{l}173 \\
(34.39)\end{array}$ & $40(40.82)$ & $\begin{array}{l}213 \\
(35.44)\end{array}$ & 0.224 & NR \\
\hline Azithromycin and Ampiclox & $\begin{array}{l}1 \\
(0.20)\end{array}$ & $0(0.00)$ & $\begin{array}{l}1 \\
(0.17)\end{array}$ & 0.659 & $N R+N R$ \\
\hline Azithromycin and Cefalexin & $\begin{array}{l}1 \\
(0.20)\end{array}$ & $0(0.00)$ & $\begin{array}{l}1 \\
(0.17)\end{array}$ & 0.656 & $\mathrm{NR}+1^{\text {st }}$ Line \\
\hline Azithromycin and Cefixime & $\begin{array}{l}1 \\
(0.20)\end{array}$ & $0(0.00)$ & $\begin{array}{l}1 \\
(0.17)\end{array}$ & 0.656 & $N R+N R$ \\
\hline Azithromycin and Ciplofloxacillin & $\begin{array}{l}2 \\
(0.40)\end{array}$ & $0(0.00)$ & $\begin{array}{l}2 \\
(0.33)\end{array}$ & 0.532 & $\mathrm{NR}+2^{\text {nd }}$ Line \\
\hline Azithromycin and Doxycycline & $\begin{array}{l}5 \\
(0.99)\end{array}$ & $0(0.00)$ & $\begin{array}{l}5 \\
(0.83)\end{array}$ & 0.322 & $N R+N R$ \\
\hline Azithromycin and Metronidazole & $\begin{array}{l}4 \\
(0.80)\end{array}$ & $0(0.00)$ & $4(0.67)$ & 0.376 & $N R+N R$ \\
\hline Azithromycin and Nitrofurantoin & $\begin{array}{l}1 \\
(0.20)\end{array}$ & $0(0.00)$ & $\begin{array}{l}1 \\
(0.17)\end{array}$ & 0.659 & $\mathrm{NR}+1^{\text {st }}$ Line \\
\hline Cefadroxil capsule & $\begin{array}{l}1 \\
(0.20)\end{array}$ & $0(0.00)$ & $\begin{array}{l}1 \\
(0.17)\end{array}$ & 0.659 & NR \\
\hline Cefixime & $\begin{array}{l}1 \\
(0.20)\end{array}$ & $0(0.00)$ & $\begin{array}{l}1 \\
(0.17)\end{array}$ & 0.659 & NR \\
\hline Ceftriaxone & $\begin{array}{l}2 \\
(0.40)\end{array}$ & $0(0.00)$ & $\begin{array}{l}2 \\
(0.33)\end{array}$ & 0.532 & NR \\
\hline Cephalexin & $\begin{array}{l}56 \\
(11.13)\end{array}$ & 15 (15.31) & $\begin{array}{l}71 \\
(11.81)\end{array}$ & 0.236 & $1^{\text {st }}$ Line \\
\hline
\end{tabular}




\begin{tabular}{|c|c|c|c|c|c|}
\hline $\begin{array}{l}\text { Chloramphenicol and } \\
\text { Metronidazole }\end{array}$ & $\begin{array}{l}1 \\
(0.20)\end{array}$ & $0(0.00)$ & $\begin{array}{l}1 \\
(0.17)\end{array}$ & 0.659 & $N R+N R$ \\
\hline Ciprofloxacin & $\begin{array}{l}110 \\
(21.87)\end{array}$ & $13(13.27)$ & $\begin{array}{l}123 \\
(20.47)\end{array}$ & 0.053 & $2^{\text {nd }}$ Line \\
\hline Ciprofloxacin and Doxycycline & $\begin{array}{l}23 \\
(4.47)\end{array}$ & $2(2.04)$ & $\begin{array}{l}25 \\
(4.16)\end{array}$ & 0.251 & $2^{\text {nd }}$ Line $+N R$ \\
\hline Clarithromycin & $\begin{array}{l}1 \\
(0.20)\end{array}$ & $1(1.02)$ & $\begin{array}{l}2 \\
(0.33)\end{array}$ & 0.196 & NR \\
\hline Doxycycline * & $\begin{array}{l}45 \\
(8.95)\end{array}$ & $2(2.04)$ & $\begin{array}{l}47 \\
(7.82)\end{array}$ & 0.020 & NR \\
\hline Doxycycline and Metronidazole & $\begin{array}{l}2 \\
(0.40)\end{array}$ & $1(1.02)$ & $\begin{array}{l}3 \\
(0.33)\end{array}$ & 0.424 & $N R+N R$ \\
\hline $\begin{array}{l}\text { Doxycycline and } \\
\text { Trimethoprim/Sulfamethoxazole }\end{array}$ & $\begin{array}{l}1 \\
(0.20)\end{array}$ & $0(0.00)$ & $\begin{array}{l}1 \\
(0.17)\end{array}$ & 0.659 & $N R+N R$ \\
\hline Flucamox & $\begin{array}{l}6 \\
(1.19)\end{array}$ & $1(1.02)$ & $\begin{array}{l}7 \\
(1.16)\end{array}$ & 0.884 & NR \\
\hline Levofloxacin & $\begin{array}{l}6 \\
(1.19)\end{array}$ & $5(5.10)$ & $\begin{array}{l}11 \\
(1.83)\end{array}$ & 0.008 & $2^{\text {nd }}$ Line \\
\hline Metronidazole & $\begin{array}{l}5 \\
(0.99)\end{array}$ & $0(0.00)$ & $\begin{array}{l}5 \\
(0.83)\end{array}$ & 0.322 & NR \\
\hline Moxifloxacin & $\begin{array}{l}0 \\
(0.00)\end{array}$ & $1(1.02)$ & $\begin{array}{l}1 \\
(0.17)\end{array}$ & 0.659 & NR \\
\hline Nitrofurantoin & $\begin{array}{l}4 \\
(0.80)\end{array}$ & $3(3.06)$ & $\begin{array}{l}7 \\
(1.16)\end{array}$ & 0.056 & $1^{\text {st }}$ Line \\
\hline Norfloxacillin & $\begin{array}{l}5 \\
(0.99)\end{array}$ & $0(0.00)$ & $\begin{array}{l}5 \\
(0.83)\end{array}$ & 0.322 & NR \\
\hline Tetracycline & $\begin{array}{l}1 \\
(0.20)\end{array}$ & $0(0.00)$ & $\begin{array}{l}1 \\
(0.17)\end{array}$ & 0.659 & NR \\
\hline Trimethoprim/Sulfamethoxazole* & $\begin{array}{l}14 \\
(2.78)\end{array}$ & $0(0.00)$ & $\begin{array}{l}14 \\
(2.33)\end{array}$ & 0.095 & $1^{\text {st }}$ Line \\
\hline total & $\begin{array}{l}503 \\
(83.69)\end{array}$ & $98(16.31)$ & $\begin{array}{l}601 \\
(100)\end{array}$ & & \\
\hline
\end{tabular}

* Drugs that ADDOs are permitted to stock. ADDOs are not mandated to stock and sell the other drugs listed

Across the wide variety of drugs that sellers suggested, we analysed whether there were any significant differences in those recommended by gender of the MC. Overall, more women (47.13\%) than men $(41.86 \%)$ were recommended a drug listed in the TSTGs as suitable for the treatment of UTIs (see table 5). The most commonly suggested antimicrobial, azithromycin, was recommended to more male clients 
(36.1\%) than female (33.1) (p-value 0.000 ) as indicated in table 5 bellow. Similarly, a combination of 'ciprofloxacin and doxycycline' was suggested to more male (5.0\%) than female clients $(0.1 \%)$ (p-value of 0.040) (see table 4). Neither azithromycin nor ciprofloxacin and doxycycline are recommended in the TSTGs for UTI treatment. Finally, $29.76 \%$ of women were recommended a $2^{\text {nd }}$ or $3^{\text {rd }}$ line drug (whether or not suitable for UTI treatment) compared to $30.83 \%$ of men (see table 5 )

Table 5 List of antimicrobials sellers were willing to dispense by clients' gender 


\begin{tabular}{|c|c|c|c|c|c|}
\hline Suggested Drug (s) & $\begin{array}{l}\text { Female's } \\
\text { Clients } \\
\mathrm{n}-121 \\
\mathrm{n}(\%)\end{array}$ & $\begin{array}{l}\text { Male's } \\
\text { Clients } \\
\text { (Total n- } \\
480) \\
\mathrm{n}(\%)\end{array}$ & $\begin{array}{l}\text { Overall } \\
\text { n-601 } \\
n(\%)\end{array}$ & $\begin{array}{l}\text { P- } \\
\text { Value }\end{array}$ & $\begin{array}{l}\text { Not } \\
\text { Recommended for } \\
\text { UTI (NRUTI) } \\
\text { \&Treatment Line }\end{array}$ \\
\hline Amoxicillin & $4(3.31)$ & $5(1.04)$ & $\begin{array}{l}9 \\
(1.50)\end{array}$ & 0.067 & $1^{\text {st }}$ Line \\
\hline Amoxicillin and metronidazole & $0(0.00)$ & $2(0.42)$ & $\begin{array}{l}2 \\
(0.33)\end{array}$ & 0.477 & $1^{\text {st }}$ Line+ NRUTI \\
\hline Amoxicillin/clavulanic Acid & $7(5.79)$ & 16(3.33) & $\begin{array}{l}23 \\
(3.83)\end{array}$ & 0.209 & $3^{\text {rd }}$ line \\
\hline Ampicillin & $\begin{array}{l}0 \\
(0.00)\end{array}$ & $1(0.21)$ & $\begin{array}{l}1 \\
(0.17)\end{array}$ & 0.615 & NRUTI \\
\hline Ampiclox & $2(1.65)$ & $7(1.46)$ & $\begin{array}{l}1 \\
(0.17)\end{array}$ & 0.875 & NRUTI \\
\hline Azithromycin & $\begin{array}{l}40 \\
(33.06)\end{array}$ & $173(36.04)$ & $\begin{array}{l}213 \\
(35.44)\end{array}$ & 0.000 & NRUTI \\
\hline Azithromycin and Ampiclox & $1(0.83)$ & $0(0.00)$ & $\begin{array}{l}1 \\
(0.17)\end{array}$ & 0.046 & NRUTI + NRUTI \\
\hline Azithromycin and Cephalexin & $1(0.83)$ & $0(0.00)$ & $\begin{array}{l}1 \\
(0.17)\end{array}$ & 0.046 & NRUTI $+1^{\text {st }}$ Line \\
\hline Azithromycin and Cefixime & $0(0.00)$ & $1(0.21)$ & $\begin{array}{l}1 \\
(0.17)\end{array}$ & 0.615 & NRUTI + NRUTI \\
\hline Azithromycin and Ciplofloxacillin & $0(0.00)$ & $2(0.42)$ & $\begin{array}{l}2 \\
(0.33)\end{array}$ & 0.477 & NRUTI $+2^{\text {nd }}$ Line \\
\hline Azithromycin and Doxycycline & $1(0.83)$ & $4(0.83)$ & $\begin{array}{l}5 \\
(0.83)\end{array}$ & 0.994 & NRUTI + NRUTI \\
\hline Azithromycin and Metronidazole & $0(0.00)$ & $4(0.83)$ & $4(0.67)$ & 0.314 & NRUTI + NRUTI \\
\hline Azithromycin and Nitrofurantoin & $0(0.00)$ & $1(0.21)$ & $\begin{array}{l}1 \\
(0.17)\end{array}$ & 0.615 & NRUTI+ $1^{\text {st }}$ Line \\
\hline Cefadroxil capsule & $0(0.00)$ & $1(0.21)$ & $\begin{array}{l}1 \\
(0.17)\end{array}$ & 0.615 & NRUTI \\
\hline Cefixime & $0(0.00)$ & $1(0.21)$ & $\begin{array}{l}1 \\
(0.17)\end{array}$ & 0.615 & NRUTI \\
\hline Ceftriaxone & $1(0.85)$ & $1(0.21)$ & $\begin{array}{l}2 \\
(0.33)\end{array}$ & 0.291 & NRUTI \\
\hline Cephalexin & $\begin{array}{l}11 \\
(9.09)\end{array}$ & $60(12.50)$ & $\begin{array}{l}71 \\
(11.81)\end{array}$ & 0.143 & $1^{\text {st }}$ Line \\
\hline
\end{tabular}




\begin{tabular}{|c|c|c|c|c|c|}
\hline $\begin{array}{l}\text { Chloramphenicol and } \\
\text { Metronidazole }\end{array}$ & $0(0.00)$ & $1 \quad(0.21)$ & $\begin{array}{l}1 \\
(0.17)\end{array}$ & 0.615 & NRUTI+ NRUTI \\
\hline Ciprofloxacin & $\begin{array}{l}28 \\
(23.14)\end{array}$ & $95(19.79)$ & $\begin{array}{l}123 \\
(20.47)\end{array}$ & 0.415 & $2^{\text {nd }}$ Line \\
\hline Ciprofloxacin and Doxycycline & $1(0.83)$ & $24(5.00)$ & $\begin{array}{l}25 \\
(4.16)\end{array}$ & 0.040 & $2^{\text {nd }}$ Line + NRUTI \\
\hline Clarithromycin & $1(0.83)$ & $1(0.21)$ & $\begin{array}{l}2 \\
(0.33)\end{array}$ & 0.291 & NRUTI \\
\hline Doxycycline & $9(7.44)$ & $38(7.92)$ & $\begin{array}{l}47 \\
(7.82)\end{array}$ & 0.861 & NRUTI \\
\hline Doxycycline and Metronidazole & $0(0.00)$ & $3 \quad(0.62)$ & $\begin{array}{l}3 \\
(0.33)\end{array}$ & 0.383 & NRUTI+ NRUTI \\
\hline $\begin{array}{l}\text { Doxycycline and } \\
\text { Trimethoprim/Sulfamethoxazole }\end{array}$ & $0(0.00)$ & $1(0.21)$ & $\begin{array}{l}1 \\
(0.17)\end{array}$ & 0.615 & NRUTI+ NRUTI \\
\hline Flucamox & $1(0.83)$ & $6(1.25)$ & $\begin{array}{l}7 \\
(1.16)\end{array}$ & 0.698 & NRUTI \\
\hline Levofloxacin & $0(0.00)$ & $11(2.29)$ & $\begin{array}{l}11 \\
(1.83)\end{array}$ & 0.093 & $2^{\text {nd }}$ Line \\
\hline Metronidazole & $2(1.65)$ & $3(0.62)$ & $\begin{array}{l}5 \\
(0.83)\end{array}$ & 0.266 & NRUTI \\
\hline Moxifloxacin & $0(0.00)$ & $1(0.21)$ & $\begin{array}{l}1 \\
(0.17)\end{array}$ & 0.615 & NRUTI \\
\hline Nitrofurantoin & $3(2.48)$ & $4(0.83)$ & $\begin{array}{l}7 \\
(1.16)\end{array}$ & 0.132 & $1^{\text {st }}$ Line \\
\hline Norfloxacillin & $2(1.65)$ & $3(0.62)$ & $\begin{array}{l}5 \\
(0.83)\end{array}$ & 0.266 & NRUTI \\
\hline Tetracycline & $1(0.83)$ & $0(0.00)$ & $\begin{array}{l}1 \\
(0.17)\end{array}$ & 0.046 & NRUTI \\
\hline Trimethoprim/Sulfamethoxazole & $4(3.32)$ & $10(2.08)$ & $\begin{array}{l}14 \\
(2.33)\end{array}$ & 0.426 & $1^{\text {st }}$ Line \\
\hline total & $\begin{array}{l}121 \\
(20.13)\end{array}$ & $\begin{array}{l}480 \\
(79.87)\end{array}$ & $\begin{array}{l}601 \\
(100)\end{array}$ & & \\
\hline
\end{tabular}

\section{Discussion}

As part of a wider, two phase, three-country investigation of the drivers of AMR in East Africa, this study aimed to determine the role played by private drug shops in the ABs provision landscape in three sites in Tanzania (Mwanza, Mbeya and Kilimanjaro regions). Tanzania's pharmacy (prescription handling and control) regulations prohibit the dispensing of ABs without prescription [19]. We investigated how different kinds of drug sellers, in different contexts, would respond to a client who did not have a 
prescription, reporting symptoms of UTI and only if questioned, a history of similar symptoms and selfmedication

The results of this survey suggest that compliance with certain areas of drug provider regulation is relatively good; most premises displayed certificates of qualification and were in at least adequate condition to store and sell medications, and only a small minority may have been staffed by personnel below the minimum required age. Furthermore, the study records relatively good client/seller interaction scores compared to phase 1 of our study (in which MCs made a straightforward request for a specific drug - amoxicillin, without necessarily describing UTI-like symptoms). In that phase interaction seemed poor and transactional, with only $10.6 \%$ addressing $1-3$ clinically relevant questions or treatment suggestions on our 8-point scale and only 1.2\% addressing between 4-7 [16]. By comparison in phase 2, reported here, when presented with symptoms and a request for advice, $90 \%$ of sellers addressed between 1 and 3 clinically relevant questions/advice items and 10\% scored between 4 and 7 . Similarly, on the critically important question 'do you have a prescription', scores were better in response to scenario 2 /phase 2 but only marginally so $(6.1 \%$ compared to $1.2 \%$ in scenario 1$)$.

However, notwithstanding the improved 'quality of interaction' in response to a client's request for advice rather than demand for a named drug, these scores were dominated by questions related to patients' illness history: 'have you experienced these symptoms before' (42.3\%), and to a lesser extent: 'are you taking other medication' (14.0\%). Meanwhile, propensity to ask the questions and give the advice most relevant to both regulation and AMR stewardship remained worryingly low ('do you have a prescription' $6.1 \%$, 'have you seen a doctor' $17.9 \%$, 'you should see a doctor/get a prescription' $12.1 \%$, 'you should take or finish a whole course' $9.4 \%$ ). This is particularly noteworthy given that all sellers are officially mandated to sell antibiotics only if a prescription is provided. While the pharmacy sector performed better than ADDO level sellers, two thirds of pharmacies still failed to address these most clinically/AMR relevant questions in our 8-point checklist. Concern that improved interaction does not necessarily indicate improved antibiotic stewardship seem to be confirmed by the fact that most sellers $(89.7 \% \mathrm{Cl}$ : $87.2 \%-91.8 \%$ with little difference between the sectors), dispensed an antimicrobial OTC despite clients not being in possession of a prescription (only slightly fewer than the $94.6 \%$ who dispensed amoxicillin in response to a direct request in phase 1 of the study [16]).

Our findings corroborate those of another study conducted in the Moshi urban district of Kilimanjaro region which found that $92 \%$ of pharmacies dispensed antimicrobials to clients without prescription [23], but do so for a larger sample in a larger number of areas of Tanzania. Furthermore, this study went beyond assessing if sellers would dispense without prescription and in addition investigated whether sellers would sell less than the minimum course mandated by the TSTGs. We found that $58.9 \%$ of sellers (with little difference between pharmacy and ADDO sectors) were willing to dispense 'just a few days' worth' of a drug. This is less than the $93.1 \%$ who sold a 'half course' in phase 1 but remains a dangerously high figure given the possible association between under dosing and the development of AMR. 
In line with a previous study in Moshi urban [23], the current study also found that azithromycin was the drug most commonly suggested for UTI even though it is not recommended for UTI in the TSGT. Proportionately, more pharmacies than ADDOs inappropriately suggested azithromycin as a UTI treatment $(40,40.8 \%$ vs $173,34.4 \%)$ but far more ADDOs than pharmacies suggested doxycycline inappropriately $(45,8.9 \%$ vs $2,2.0 \%)$. This drug is also not recommended for the treatment of UTI but it is one which ADDOs are mandated to sell.

Antibiotic drugs are classified into three 'lines' according to their effectiveness. Second- and third-line drugs are more effective than first-line, but also more toxic with more potential side effects. They should be held in reserve for cases where first-line drugs have failed to clear an infection and/or the pathogen has become resistant to a first- or second-line drug [24]. If the seller asked about illness history, our scenario included a report that the MC had taken amoxicillin, but it 'had not worked'. At one level it is unsurprising then that only 9 (1.8\%) ADDOs sellers and no pharmacists suggested amoxicillin. However, it should be remembered that only 284 (42,3\%) asked about illness history and only 94 (14\%) about other medication. From this perspective the failure to suggest amoxicillin is noteworthy, as is the fact that only 101 (16.8\%) recommended a first line drug for UTI (which included amoxicillin [9, 1.5\%], cephalexin [71, $11.81 \%]$, trimethoprim/Sulfamethoxazole [14, 2.3\%] and nitrofurantoin $[7,1.16])$.

Some 134 sellers (35.44\% of all types) recommended a second line drug (which included ciprofloxacin $[123,20.5 \%]$ and levofloxacin $[11,1.8 \%])$, with a very small number recommending the third-line drug amoxiclav (23,3.83\%). Meanwhile, 290 (49\%) sellers suggested antimicrobials that the TSTGs classify as inappropriate for the treatment of UTI. The dispensing of drugs inappropriate to symptoms described suggests that sellers either lack familiarity with the TSTGs, or neglect them, or customize them (e.g., ADDOs selling a drug which is not suitable to treat the symptoms described by a client but which they are mandated to dispense). It also reinforces that empirical diagnosis of symptoms is a specialist skill which explains why antibiotics should not be dispensed without a doctor's prescription.

While our scenario together with sellers' relatively high propensity to ask about 'illness history' and 'other medication' might explain some of the failure to dispense Amoxicillin, they do not explain why sellers did not recommend the alternative 1st line treatment they are empowered to dispense. Furthermore, even though our methodology meant that if offered amoxicillin, our MCs requested 'something stronger', because 'amoxicillin had not worked', a thorough questioning of the client would have revealed that they had previously taken 'only a few days' worth' of the drug. Results from phase 1 of our study suggests that sellers know that clients habitually take a 'half course' because sellers habitually dispense less than the recommended minimum courses. Therefore, not only did ADDO sellers not have permission to sell second-line drugs, but both they and the pharmacy sellers might reasonably have advised MCs to buy and finish a full course of an appropriate first-line drug before second-line drugs were even considered.

\section{Strengths and limitations of the study}

This study has several methodological strengths: Firstly, the mystery client method allows for more objective assessment of dispensing practice than would self-reported responses to a questionnaire. 
Secondly, the scenario used allowed us to assess all of the following simultaneously: compliance with general standards, quality of seller/client interactions, range of drugs dispensed in response to a common set of symptoms, and whether sellers would sell antibiotics without a prescription and do so in quantities less than the recommended course. It also allowed us to compare results to an earlier scenario which was based on a simple request for a commonly dispensed drug. Thirdly, the study had a larger sample size and covered more regions than earlier Tanzanian studies.

Limitations of the study were: While more extensive than previous studies in this region, the sample size in this study was only a subset of the $100 \%$ sample in our own phase 1 study of the same regions, and like it, is limited in scope to 3 regions of Tanzania. Secondly, our quantitative methods demonstrate patterns of behaviour but do not necessarily give us the drivers for poor practices, which calls for a qualitative approach. Thirdly, the drug outlets were mostly located in urban settings and the sample is dominated by findings from Mwanza.

\section{Conclusion}

In Kilimanjaro, Mbeya and Mwanza regions, Tanzania, this study tested pharmacy and ADDO drug seller responses to mystery clients who presented with symptoms of UTI and asked for advice and revealed only if questioned that they had no prescription and had previously self-medicated. Its findings have implications for the treatment of UTI, the promotion of antibiotic stewardship and for regulatory policy. The study identified, first: a widespread willingness to dispense antibiotics without prescription, and in quantities below the recommended minimum course: Second, that less than half the drugs recommended were listed in the Tanzanian Standard Treatment Guidelines (TSTGs) as suitable for the treatment of UTI: Third, that ADDOs frequently stocked antimicrobials they were not permitted to sell, including 2nd and 3rd line/watch list drugs, and that sellers of all types too readily bypassed the $1 \mathrm{st}$ line/access treatment for UTI, amoxicillin, without rigorously questioning whether clients had previously used it or used it appropriately: Fourth, that while pharmacies questioned clients more effectively than ADDOs the sector still underperformed, especially in relation to questions relevant to antibiotic stewardship: Fifth, that while women were both more thoroughly questioned and more likely to have a relevant drug recommended, like men, the majority were offered drugs not recommended for treating UTI in the TSTGs.

Tanzania was the first African country WHO recognized for achieving a functioning regulatory system for medical products [14], yet this study demonstrates a lack of compliance with some of the most important elements of regulation. Recurrent inspection is required to enforce existing regulations on authorized medicines and prescriptions. However, this should be accompanied by continuing professional development that reinforces the importance of WHO AWaRE categories and national standard treatment guidelines on appropriate drugs and minimum course, and which encourages sellers to question and advise clients more thoroughly. Community pharmacies and ADDO sellers need to be encouraged to recognize their role in ensuring the health of their customers and communities. Further qualitative study is required to identify sellers' perceptions of their current dispensing practices and explore what might motivate their compliance with regulation and improve their stewardship of life saving antimicrobials. 


\section{Abbreviations}

ABs

Antibiotics

ADDO

Accredited Drug Dispensing Outlets

AMR

Antimicrobial Resistance

DDDs

Daily Defined Doses

GPS

Global Positioning System

HATUA

Holistic Approach to Unravel Antibacterial Resistance in East Africa

LMICs

Low and Middle Income Countries

MCs

Mystery Clients

OTC

Over the Counter

TMDA

Tanzania Medicine \& Medical Devices Authority

TSTG

Tanzania Standard Treatment Guideline

UTI

Urinary Tract Infection

\section{Declarations}

\section{Ethics approval and concert to participate}

All methods were performed in accordance with relevant guidelines and regulations. The study received ethical approval from the University of St Andrews, UK (No. MD14548, 10/09/19), National Institute for Medical Research, Tanzania (No. 2831, updated 26/07/19), Mbeya Medical Research and Ethics Committee (No. SZEC-2439/R.A/V.1/30), Kilimanjaro Christian Medical College, Tanzania (No. 2293, updated 14/08/19) and Catholic University of Health and Allied Sciences /Bugando Medical Center research ethics and review committee (No. CREC /266/2018, updated on 02/2019).

Participants were not consented basing on the criteria for Institutional Ethics Research Committee (IREC) approval of research in Tanzania that researches are allowed when the risk to participants is minimal and the benefits greatly out-ways the risk [25]. The scenario did not require sellers to do or say anything 
outside of what they would otherwise be doing as part of their usual practice. Furthermore, the responses gathered at particular sites and from particular individuals, are not identifiable in the data presented.

\section{Consent to publication}

Not applicable

\section{Availability of data and materials}

The dataset generated and analyzed during the current study is available from correspond author on reasonable request.

\section{Competing Interests}

The authors declare no competing interest.

\section{Funding}

This study was funded by HATUA project. The Holistic Approach to Unravel Antibacterial Resistance in East Africa is a 3-year Global Context Consortia Award (MR/S004785/1) funded by the National Institute for Health Research, Medical Research Council and the Department of Health and Social Care. The award is also part of the EDCTP2 programme supported by the European Union. The funders had no role in study design, data collection and analysis, decision to publish or preparation of the manuscript.

\section{Authors' Contributions}

PMN participated in data analysis and preparation of the first draft of the manuscript. MFM coordinated data collection in Mwanza, Mbeya and Kilimanjaro, and participated in data analysis and manuscript preparation, JRM contributed to the conceptualization of the project, co-designed the tools and supervised the data collection. ETK did the data analysis and helped in writing the first draft of the manuscript. SM did the data analysis and helped in writing the first draft of the manuscript. MWM did the data analysis and helped in writing the first draft of the manuscript. SMM contributed in reviewing the manuscript. NEN coordinated data collection in Mbeya. BTM contributed to the conceptualization of the project and coordinated data collection in Kilimanjaro. KK, MK, F.B.-P and WS contributed to the conceptualization of the project, co-designed the tools and co-wrote the final manuscript. AS contributed to the conceptualization, coordinated data collection and reviewed the manuscript. MTGH led the conceptualization of the project, designed the protocol and was the guarantor of the project. SEM contributed to the conceptualization of the project, co-designed the tools, performed data analysis, reviewed the manuscript and coordinated data collection in Mwanza, Mbeya and Kilimanjaro.

\section{Acknowledgments}

We thank the assistance given by Mwanza, Mbeya and Kilimanjaro administrative Secretaries in implementing this study. In addition, we thank CUHAS $20194^{\text {th }}$ year Bachelor of Pharmacy students who 
acted as MCs. Furthermore, we would like to acknowledge the contribution of all members of the HATUA Consortium. HATUA Consortium: Members included above in list of authorship plus: Catherine Kansiime, Arun Gonzales Decano, Dominique L Green, John Mwaniki, Benon Asiimwe, John Kiiru, Derek Sloan and Joel Bazira

\section{References}

1. Sengupta S, Chattopadhyay MK, Grossart H-P: The multifaceted roles of antibiotics and antibiotic resistance in nature. Frontiers in microbiology 2013, 4:47.

2. Hughes $D$, Karlén A: Discovery and preclinical development of new antibiotics. Upsala Journal of Medical Sciences 2014, 119(2):162-169.

3. Klein EY, Van Boeckel TP, Martinez EM, Pant S, Gandra S, Levin SA, Goossens H, Laxminarayan R: Global increase and geographic convergence in antibiotic consumption between 2000 and 2015. Proceedings of the National Academy of Sciences 2018, 115(15):E3463-E3470.

4. Bell BG, Schellevis F, Stobberingh E, Goossens $H$, Pringle M: A systematic review and meta-analysis of the effects of antibiotic consumption on antibiotic resistance. BMC infectious diseases 2014, 14(1):1-25.

5. O'neill J: Antimicrobial resistance. Tackling a crisis for the health and wealth of nations 2014.

6. Founou RC, Founou LL, Essack SY: Clinical and economic impact of antibiotic resistance in developing countries: a systematic review and meta-analysis. PloS one 2017, 12(12):e0189621.

7. Laxminarayan R, Duse A, Wattal C, Zaidi AK, Wertheim HF, Sumpradit N, Vlieghe E, Hara GL, Gould IM, Goossens $\mathrm{H}$ : Antibiotic resistance-the need for global solutions. The Lancet infectious diseases 2013, 13(12):1057-1098.

8. Belachew SA, Hall L, Selvey LA: Non-prescription dispensing of antibiotic agents among community drug retail outlets in Sub-Saharan African countries: a systematic review and meta-analysis. Antimicrobial Resistance \& Infection Control 2021, 10(1):1-15.

9. Prestinaci F, Pezzotti P, Pantosti A: Antimicrobial resistance: a global multifaceted phenomenon. Pathogens and global health 2015, 109(7):309-318.

10. Cars 0 , Nordberg P: Antibiotic resistance-The faceless threat. International Journal of Risk \& Safety in Medicine 2005, 17(3, 4):103-110.

11. Istúriz RE, Carbon C: Antibiotic use in developing countries. Infection Control \& Hospital Epidemiology 2000, 21(6):394-397.

12. Chokshi A, Sifri Z, Cennimo D, Horng H: Global contributors to antibiotic resistance. Journal of global infectious diseases 2019, 11(1):36.

13. Schellack N, Benjamin D, Brink A, Duse A, Faure K, Goff D, Mendelson M, Meyer J, Miot J, Perovic O: A situational analysis of current antimicrobial governance, regulation, and utilization in South Africa. International journal of infectious diseases 2017, 64:100-106. 
14. Van Boeckel TP, Gandra S, Ashok A, Caudron Q, Grenfell BT, Levin SA, Laxminarayan R: Global antibiotic consumption 2000 to 2010: an analysis of national pharmaceutical sales data. The Lancet infectious diseases 2014, 14(8):742-750.

15. Asiimwe BB, Kiiru J, Mshana SE, Neema S, Keenan K, Kesby M, Mwanga JR, Sloan DJ, Mmbaga BT, Smith VA: Protocol for an interdisciplinary cross-sectional study investigating the social, biological and community-level drivers of antimicrobial resistance (AMR): Holistic Approach to Unravel Antibacterial Resistance in East Africa (HATUA). BMJ open 2021, 11(3):e041418.

16. Ndaki PM, Mushi MF, Mwanga JR, Konje ET, Ntinginya NE, Mmbaga BT, Keenan K, Sabiiti W, Kesby $\mathrm{M}$, Benitez-Paez $\mathrm{F}$ et al: Dispensing Antibiotics without Prescription at Community Pharmacies and Accredited Drug Dispensing Outlets in Tanzania: A Cross-Sectional Study. Antibiotics 2021, 10(8):1025.

17. Medicines T: Medical Devices Authority (TMDA) List of registered medicines is available at https://imis. tfda. go. tz/portal/registered-products/registered-drug-products-1. TMDA website 2019, 1.

18. Krugman S, Katz SL, Gershon AA: Infectious diseases of children. 1992.

19. Rutta E, Senauer K, Johnson K, Adeya G, Mbwasi R, Liana J, Kimatta S, Sigonda M, Alphonce E: Creating a new class of pharmaceutical services provider for underserved areas: the Tanzania accredited drug dispensing outlet experience. Progress in community health partnerships: research, education, and action 2009, 3(2):145-153.

20. Health TMo: Standard Treatment Guidelines \& National Essential Medications List: Tanzania Mainland. In.; 2017.

21. Aanensen DM, Huntley DM, Feil EJ, al-Own Fa, Spratt BG: EpiCollect: linking smartphones to web applications for epidemiology, ecology and community data collection. PloS one 2009, 4(9):e6968.

22. Aanensen DM, Huntley DM, Menegazzo M, Powell Cl, Spratt BG: EpiCollectt: linking smartphones to web applications for complex data collection projects. F1000Research 2014, 3.

23. Horumpende PG, Sonda TB, van Zwetselaar M, Antony ML, Tenu FF, Mwanziva CE, Shao ER, Mshana $\mathrm{SE}, \mathrm{Mmbaga} \mathrm{BT}$, Chilongola JO: Prescription and non-prescription antibiotic dispensing practices in part I and part II pharmacies in Moshi Municipality, Kilimanjaro Region in Tanzania: A simulated clients approach. PloS one 2018, 13(11):e0207465.

24. Sharland M, Pulcini C, Harbarth S, Zeng M, Gandra S, Mathur S, Magrini N: Classifying antibiotics in the WHO Essential Medicines List for optimal use-be AWaRe. The Lancet Infectious Diseases 2018, 18(1):18-20.

25. Committee TNHRE: Guidelines of ethics for health research in Tanzania. Tanzania National Health Research Ethics Committee: Dar es Salaam, Tanzania 2009, 2009.

\section{Supplementary Files}

This is a list of supplementary files associated with this preprint. Click to download. 
- SupplementaryinformationMCS23rdJan2022.docx

Page 26/26 